\title{
Utilisation de la thermographie IR pour l'estimation de l'évaporation régionale I. Mise au point méthodologique sur le site de la Crau
}

\author{
Bernard SEGUIN $\left({ }^{*}\right)$, Suzanne BAELZ $\left({ }^{* *}\right)$, Jean-Marie MONGET $(* *) \&$ Vincent PETIT $(* * *)$ \\ $\left(^{*}\right)$ I.N.R.A., Station de Bioclimatologie. Centre de Recherches d'Avignon, F84140 Montfavet. \\ (**) C.T.A.M.N., Ecole des Mines de Paris. Sophia Antipolis, F06500 Valbonne. \\ (***) Centre d'Informatique géologique. Ecole des Mines de Paris, F 77305 Fontainebleau.
}

\section{RÉSUMÉ}

Evaporation, Evapotranspiration,

Echelle régionale, Télédétection,

Thermographie,

Infra-rouge thermique.
L'utilisation de la thermographie IR pour l'estimation de l'évaporation régionale a été testée sur le site de la plaine de la Crau $(50 \mathrm{~km} \times 30 \mathrm{~km})$ au Nord-Ouest de Marseille. Quatre unités relativement homogènes et de dimensions suffisantes pour la télédétection par satellite (de l'ordre de 50 à $200 \mathrm{~km}^{2}$ ) y ont été délimitées, dont une en Crau sèche et trois en Crau humide. Parmi celles-ci, deux sites (Crau sèche et Crau humide) ont été choisis pour l'installation de stations de mesures microclimatiques au sol. Ces deux postes ont fonctionné en permanence pendant 3 à 4 mois au cours des étés 1978 et 1979. Ils ont permis :

- d'une part d'évaluer l'intensité des écarts microclimatiques entre les deux zones,

- d'autre part, de tester les possibilités de calcul de l'évaporation en utilisant la mesure de température de surface et de comparer les résultats obtenus à l'évaporation de référence déterminée par la méthode du rapport de BOWEN et également par des méthodes classiques approchées.

\section{SUMMARY \\ Evaporation, \\ Evapotranspiration. \\ Regional scale, \\ Remote sensing, \\ Thermography, \\ Thermal infrared.}

Use of thermal IR for estimating regional evaporation

\section{Pilot trials on the Crau plain}

The use of thermal IR for estimating regional evaporation has been tested on the Crau plain $(50 \mathrm{~km} \times 30 \mathrm{~km})$ northwest of Marseille. Within it, 4 sufficiently homogeneous units were selected, each large enough for use of satellite remote sensing (from 50 to $200 \mathrm{~km}^{2}$ ). One corresponds to the dry Crau, while the three others are located within the irrigated Crau. Two sites (dry Crau and one irrigated) were chosen for ground test studies, corresponding to microclimate and energy budget measurements continuously recorded during 3 to 4 months in the summers of 1978 and 1979.

The data obtained first allow the characterization of microclimatic differences between dry and irrigated Crau, either within a given day (fig. 4), or on a long-term basis (fig. 5) where surface temperature differences of up to $20-25^{\circ}$ may appear at midday.

The main interest of these ground data is to test the feasibility of using $T_{s}$ measurements for estimating evaporation. When based on the currently adopted energy budget method combined with aerodynamic transfer, this appears to work well for the irrigated area. But, in dry conditions, it greatly depends upon the estimate of roughness parameter $Z_{0}$ and the adoption of the Tном correction (fig. 7). On a long-term basis, the simplified approach proposed by JACKSON et al. (1977) ET $=A+R n-B\left(T_{s}-T_{a}\right)$, using one single surface temperature data, appears as satisfactory as the complete method. But one has to adopt local parameters $\mathrm{A}$ and $\mathrm{B}$, fairly different from the values originally proposed by the authors (fig. 8). The reasons for this are analysed and discussed.

Finally, comparisons with single approaches $\left(E T=R n\right.$ or $\left.E T=\frac{\Delta}{\Delta+\gamma} R_{n}\right)$ give no definitive superiority for surface temperature-based methods, since it seems to be really difficult to ensure precision better than 10 to $20 \%$. On the other hand, these last methods work equally for the two sites and give the correct differences between dry and irrigated Crau. The single methods, howcver, are only appropriate to one or the other of these conditions (fig. 9, 10,11, 12).

Use of thermal I.R. for estimating evaporation thus appears to be problematic for fixing absolute values (unless locally adapted by using known ground parameters), but really promising for evaluating quantitative spatial variations.

\section{INTRODUCTION}

L'analyse des méthodes disponibles pour l'estimation de l'évaporation ou de l'évapotranspiration à l'échelle dite « régionale " (de l'ordre de $10 \mathrm{à} 10^{5} \mathrm{~km}^{2}$ ) fait apparaître un « trou » important entre l'échelle locale ou ponctuelle (de l'ordre de l'ha) et l'échelle globale permettant d'établir des bilans approchés pour des périodes de l'ordre du mois ou de l'année et sur des surfaces allant de $10^{4}$ à $10^{6} \mathrm{~km}^{2}$ (SEGUIN, 1980).

L'apport des techniques de télédétection et, en particulier, celui des satellites permettant d'envisager l'obtention régulière d'informations aux échelles d'espace élémentaires concernées constitue un élément nouveau qui peut permet- 
tre de remédier à cet état de fait. Dans l'attente de l'approfondissement des bases d'interprétation des signaux radar, qui apparaissent comme très prometteurs pour l'estimation de l'humidité du sol, la thermographie I.R. peut être considérée comme le moyen le plus sûr d'accéder à l'estimation quantitative de l'ET en se basant sur l'utilisation classique du bilan d'énergie de la surface considérée (Seguin, 1978 ; Seguin, 1979).

La résolution des satellites utilisables dans ce domaine (de l'ordre de $0,5 \mathrm{~km}, 1 \mathrm{~km}$ et 5 à $10 \mathrm{~km}$ respectivement pour HCMM, Tiros N. \& METEOSAT), ainsi que leurs fréquences de passage $(5 \mathrm{j}, 1 \mathrm{j}$ et $1 / 2 \mathrm{~h}$ respectivement pour ces mêmes satellites) sont suffisantes pour envisager d'ores et déjà les possibilités de leur utilisation opérationnelle pour l'estimation de l'ET.

Ceci suppose, en premier lieu, la mise au point de la méthodologie utilisable, en fonction de l'ensemble des données disponibles (y compris la fiabilité et la précision des mesures par satellite) et son application à des sites expérimentaux adéquats permettant de tester les possibilités effectives de la méthode. Tel a été le but de l'expérimentation réalisée sur le site de la plaine de Crau, dans le Sud-Est de la France au voisinage Nord de Marseille. Celle-ci a rassemblé trois laboratoires (Station de Bioclimatologie I.N.R.A. Avignon, Centre Informatique géologique de l'Ecole des Mines à Fontainebleau, C.T.A.M.N. de l'Ecole des Mines à Sophia Antipolis) dans le cadre de l'action concertée «Maitrise de l'Eau en région méditerranéenne » lancée par la Délégation Générale à la Recherche Scientifique et Technique, pour la période 1979-1980. Parallèlement, le premier laboratoire cité participait au projet européen TELLUS d'utilisation du satellite HCMM, coordonné par le CCR d'Ispra (Italie) et le site de la Crau a fait partie des «tests-sites», suivis dans le cadre de ce projet.

La méthode d'utilisation de la température de surface $T_{s}$ pour l'estimation d'ET, ainsi que les bases théoriques utilisées pour aborder le problème de l'intégration spatiale à partir des points élémentaires ou «pixels » caractéristiques des satellites, ont été discutées de façon détaillée dans les publications citées plus haut. Le but de cet article est donc essentiellement de présenter les mesures effectuées sur la Crau, d'analyser et de discuter les résultats qu'elles ont permis d'obtenir et enfin d'en tirer les conclusions sur l'état actuel des possibilités. L'ensemble des données obtenues sur la Crau a fait l'objet de la présentation de 2 thèses de $3^{e}$ cycle (BAElz, 1980 ; Petit, 1980) et le lecteur intéressé peut s'y référer pour y trouver la documentation détaillée qui n'est pas contenue dans cet article de synthèse, en particulier pour la description du site expérimental et du dispositif de mesures.

\section{PRÉSENTATION BRÈVE DU SITE DE LA CRAU}

La plaine de la Crau, qui s'étend sur environ $50 \mathrm{~km}$ de long dans le sens Nord-Sud (de la mer aux Alpilles) et $30 \mathrm{~km}$ de large à l'Est du Rhône (fig. 1), a été choisie pour 3 raisons essentielles :

- le site est plat (pente régulière NE-SO de l'ordre de $2 / 1000$ ),

- il existe un fort contraste hydrique entre la Crau sèche, servant de parcours à moutons sur un sol superficiel $(20 \mathrm{à} 30 \mathrm{~cm})$ et caillouteux, et la Crau irriguée où l'apport d'eau de Durance par un système dense de canaux depuis 2 à 3 siècles a permis la constitution d'un sol limoneux

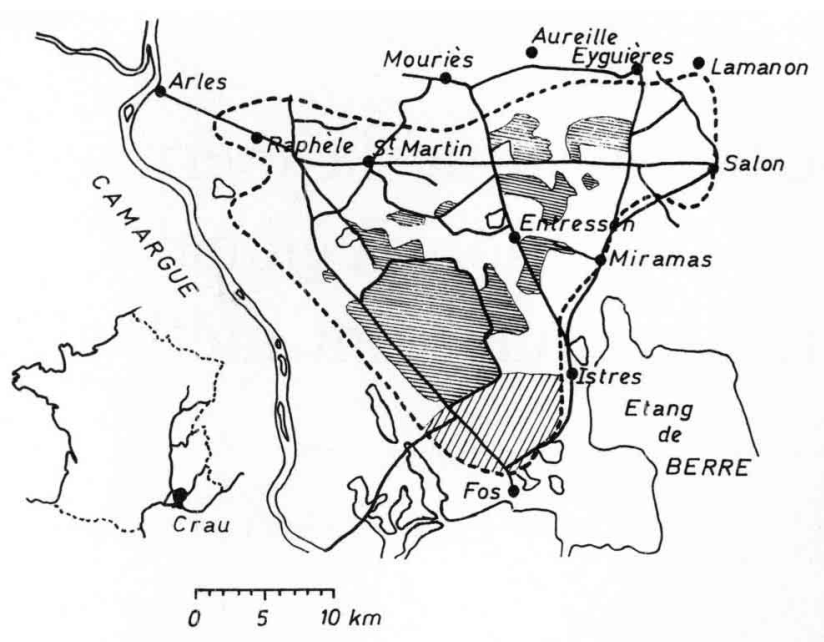

Figure 1

Cadre physique de la Crau; les zones grisées correspondent à la Crau sèche, la zone industrielle de Fos-sur-Mer est indiquée en hachuré

Physical map of the Crau plain, grey zones corresponding to the dry Crau. The industrial zone of Fos is indicated by the hatched area.

d'environ $70 \mathrm{~cm}$ de profondeur permettant la production du foin de Crau,

- ces unités contrastćes sont, par ailleurs, de dimensions suffisantes pour l'utilisation des données de satellite : la Crau sèche représente un élément homogène d'au minimum $13 \mathrm{~km} \times 13 \mathrm{~km}$, alors que les parcelles de Crau irriguées, bien qu'évidemment plus morcelées, peuvent constituer des éléments homogènes d'environ 100 ha.

L'utilisation conjuguće d'un document Landsat traité par lc GDTA et d'une carte d'occupation des sols par les cultures, établie en 1972 par la Société du Canal de Provence pour le compte de la DDA des Bouches-duRhône, a permis de délimiter 4 grandes zones qui ont été ensuite utilisées pour le traitement des données de satellite (fig. 2).

Ces zones correspondent :

- d'une part à l'unité homogène de Crau sèche 1 (environ $180 \mathrm{~km}^{2}$ ),

- d'autre part au regroupement de parcelles irriguées en 3 unités plus ou moins homogènes: la zone 2 correspond à $40 \mathrm{~km}^{2}$ autour de l'étang d'Entressen, la zone 3 à la Crau irriguée entre St-Martin de Crau et Arles $\left(100 \mathrm{~km}^{2}\right)$, la zone 4 à la partie Est constituée de prairies irriguées de plus petites dimensions, de cultures céréalières et de cultures fruitières en sec $\left(100 \mathrm{~km}^{2}\right.$ également). La proportion totale de surfaces effectivement irriguées peut être estimée respectivement à $40 \mathrm{p} .100$ pour la zone $2,70-80 \mathrm{p} .100$ pour la zone 3 et $50-60$ p. 100 pour la zone 4 .

\section{LE DISPOSITIF EXPÉRIMENTAL AU SOL}

Deux stations de mesure ont été équipées et installées dans la plaine de la Crau pour la durée de l'expérimentation par la station de Bioclimatologie I.N.R.A. d'Avignon.

Le but poursuivi était double:

- d'une part, établir à partir de mesures au sol les possibilités effectives d'estimation d'ET par l'introduction de la température radiative de surface dans les modèles de bilan d'énergie, 


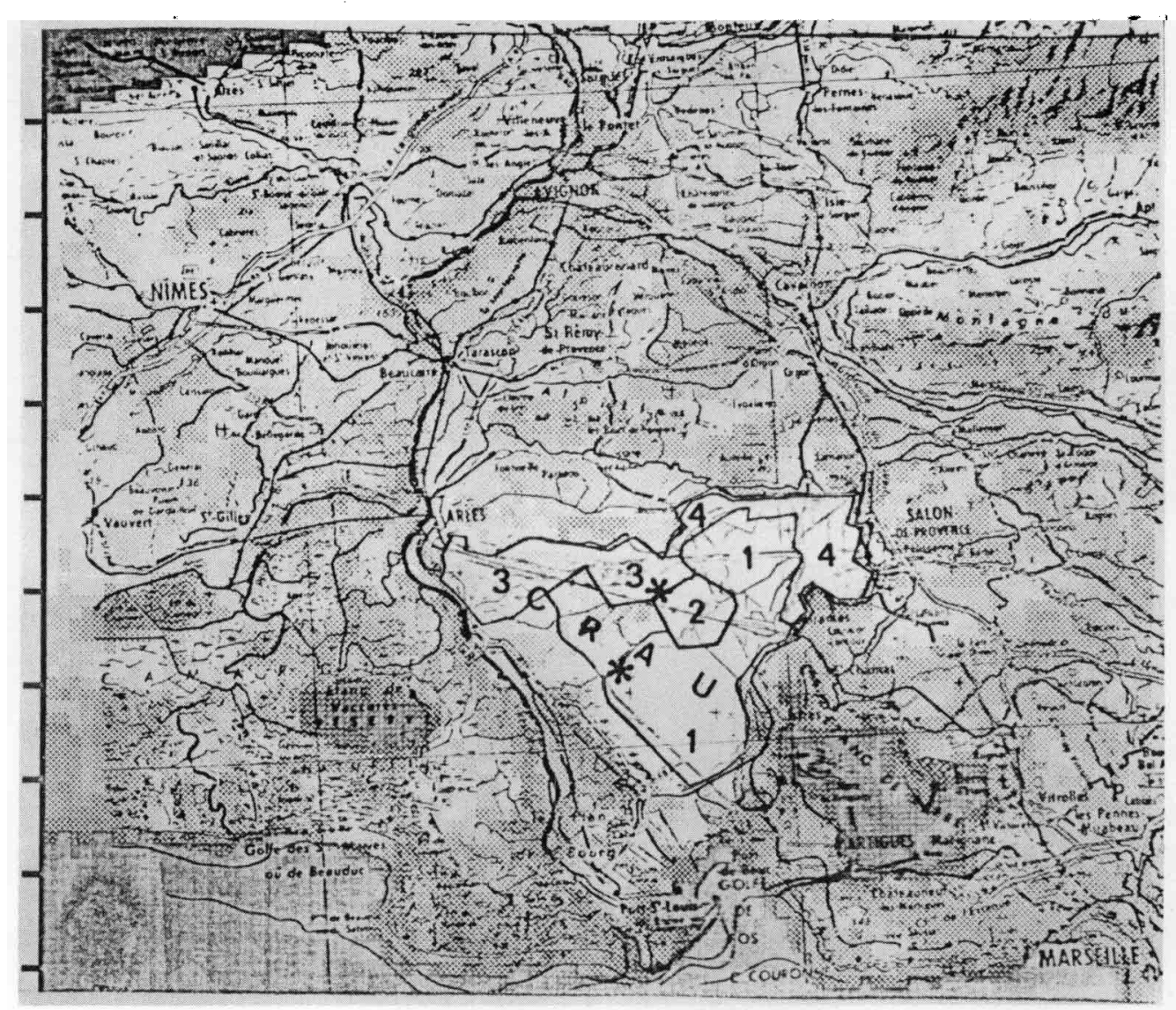

Figure 2

Délimitation des 4 zones utilisées pour l'étude.

Location of the 4 zones used in the research.

- d'autre part, utiliser la vérité terrain ainsi obtenue pour évaluer les possibilités réelles des déterminations par satellites et les conditions de leur utilisation pour l'extrapolation spatiale.

\section{A. L'équipement des stations de mesure}

Compte tenu des deux objectifs fixés plus haut, les stations de mesure comprenaient les paramètres suivants :

- le rayonnement net $\mathrm{Rn}$ par un pyrradiomètre différentiel type C.E.A.-I.N.R.A. (Ets CrouzeT),

- la température sèche et humide de l'air à $2 \mathrm{~m}$ et 0,30 m (psychromètre I.N.R.A.),

- la vitesse du vent à $2 \mathrm{~m}$ (anémomètre à vitesse instantanée I.N.R.A.),

- le flux de chaleur dans le sol S par une plaque de flux Thornthwaite à $10 \mathrm{~cm}$,

- la température du sol à $10 \mathrm{~cm}$ et $50 \mathrm{~cm}$ de profondeur.

L'ensemble de ces paramètres était recueilli, à raison d'une mesure toutes les $10 \mathrm{mn}$, sur une acquisition de données Schlumberger-Solarton 3430 et enregistré sur cassettes magnétiques, qui faisaient l'objet d'un traitement différé sur ordinateur à la Station de Bioclimatologie I.N.R.A. d'Avignon. En dehors des valeurs propres de ces paramètres, l'appareillage ainsi conçu permettrait également de déterminer l'ETR sur chaque site.

- d'une part, par la méthode du bilan d'énergie ou rapport de Bowen (PERRIER \& SEguin, 1970), dont les valeurs correspondantes $\mathrm{ET}_{\mathrm{BE}}$ seront considérées comme références,
- d'autre part, les méthodes utilisant la température de surface et dont les valeurs seront comparées à $\mathrm{ET}_{\mathrm{BE}}$.

Les 2 stations de mesure étaient implantées (fig. 3);

- l'une en Crau sèche, en bordure Nord-Est de la zone 1 ,

- l'autre en Crau irriguée, à l'extrémité Est de la zone 3, sur le domaine des Poulagères, constituant un ensemble homogène d'environ 250 ha d'un seul tenant.

Deux campagnes de mesure de longue durée ont été effectuées :

- la première au cours de l'été 1978 (juillet-octobre),

- la seconde d'avril à octobre 1979.

Du fait de défaillances momentanées, soit des appareils de mesure, soit des systèmes d'acquisition et d'enregistrement des données, l'ensemble des paramètres n'a pu être recueilli sur la totalité de ces campagnes. Après élimination des périodes où l'un au moins de ces paramètres de base est manquant, ce total de journées utilisables s'établit ainsi :

$\begin{array}{ccc} & \text { CRAU sèche } & \text { CRAU irriguée } \\ 1978 & 33 & 97 \\ 1979 & 98 & 33\end{array}$

Pour chaque année, le nombre de journées où les enregistrements sont disponibles simultanément sur les 2 zones est donc seulement de l'ordre d'une trentaine. Par contre, pour la Crau sèche en 79 et la Crau irriguée en 78 , une centaine de journées sont disponibles isolément. 


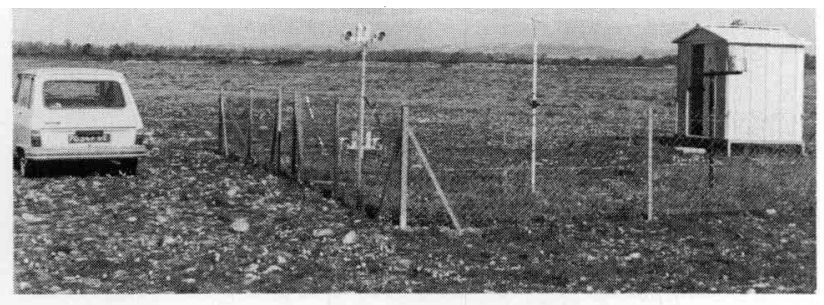

a)

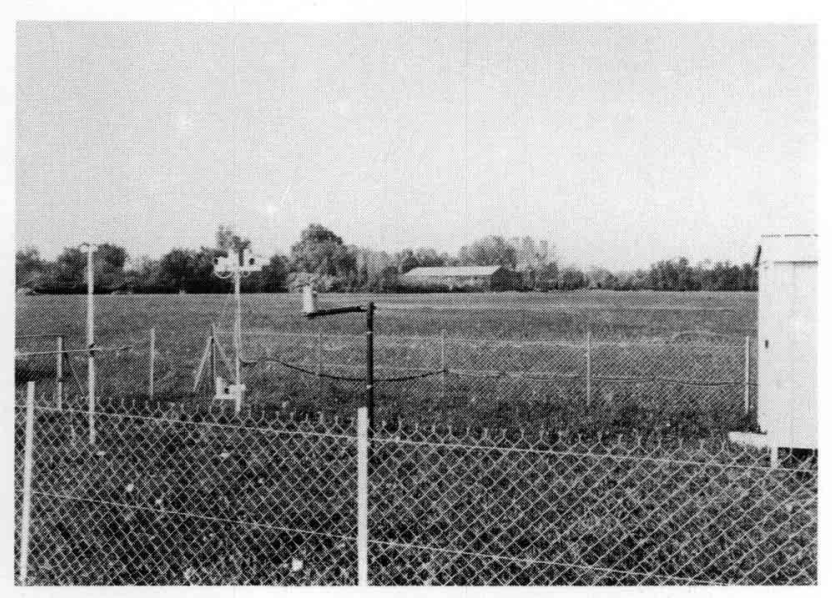

b)

Figure 3

Vues d'ensemble des stations de mesures en Crau sèche (3a) et en Crau irriguée $(3 b)$.

General views of the measurement sites in the dry Crau (3a) and the irrigated Crau ( $3 b)$.

\section{B. Caractérisation des écarts entre zone sèche et zone irriguée}

De façon générale et conformément à ce qu'on pouvait attendre, la zone sèche apparaît nettement plus chaude de jour que la zone irriguée. L'écart de nuit est inversé, mais

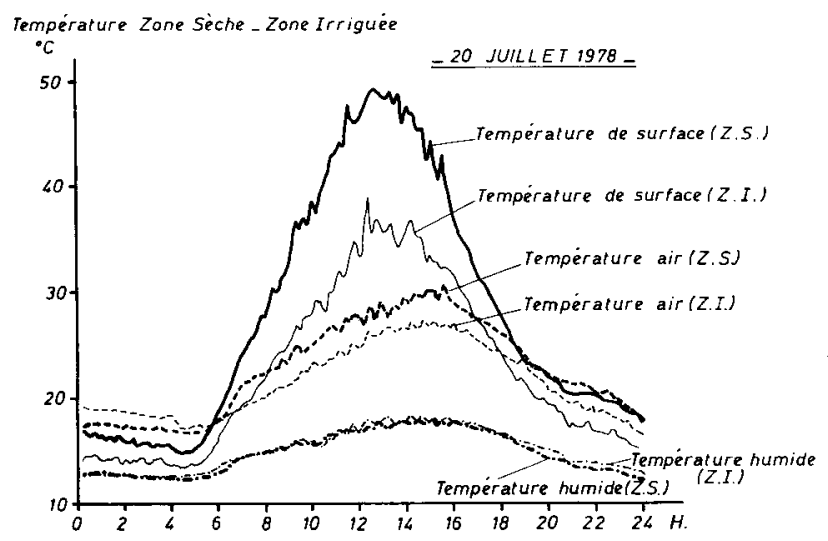

Figure 4

Evolution des températures de surface et de l'air (sèche et humide) sur les deux zones de Crau le 20 juillet 1978.

Diurnal variation of surface and air (dry and wet) temperatures on the two sites of Crau for a given day (Z.S. for dry Crau - Z.I. for irrigated Crau). (a)
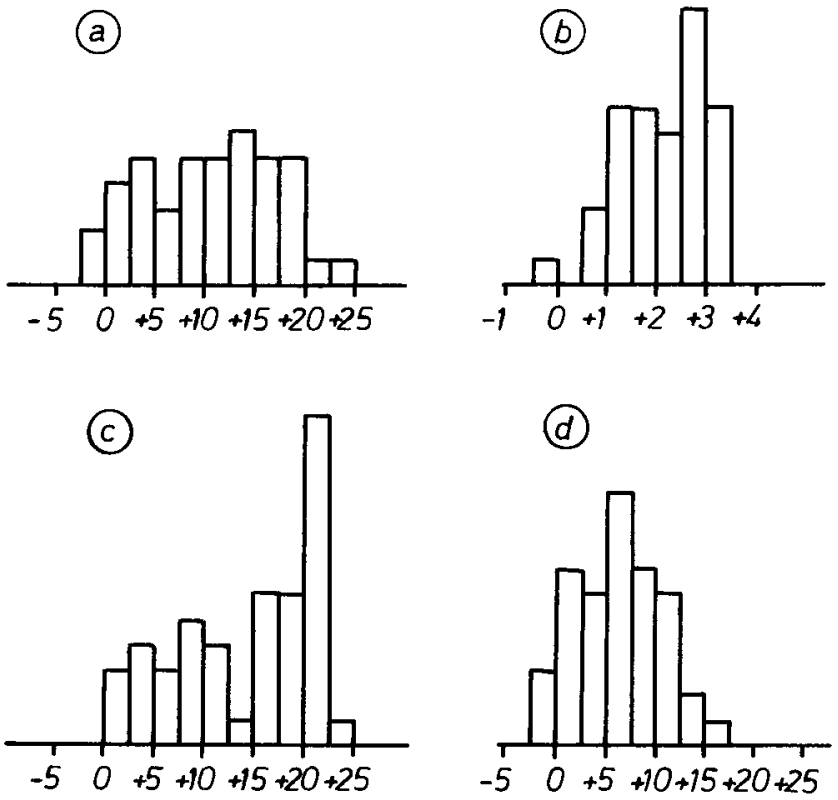

Figure 5

Histogrammes de fréquences relatives des écarts de température:

a) de la surface entre Z.S. et Z.I.

b) de l'air entre Z.S. et Z.I.

c) entre la surface et l'air $\left(T_{s}-T_{a}\right)$ en Z.S.

d) entre la surface et l'air $\left(T_{s}-T_{a}\right)$ en $Z . I$.

Relative frequency histograms of temperature differences:

a) of $T_{s}$ between dry and irrigated zones.

b) of $T_{a}$ between dry and irrigated zones.

c) $T_{5}-T_{\text {an }}$ in the dry zone.

d) $T_{s}-T_{a}$ in the irrigated zone.

ne dépasse guère les 1 à $2^{\circ}$, alors que celui de jour peut atteindre 15 à $20^{\circ}$ au moment du maximum.

C'est ce qu'indiquent les figures 4 (portant sur une journée particulière et 5 (portant sur un ensemble de 41 journées de l'été 1978, à 13 h TU).

Par ailleurs, bien qu'il soit difficile de comparer les valeurs absolues d'ETR des 2 sites (l'ETP Penman est, en général, plus élevée en zone sèche qui est pleinement dégagée alors que la zone irriguée est, en partie, abritée par un réseau de brise-vent situé plus au Nord), l'évolution du rapport $\frac{\text { ETR }}{\text { ETP }}$ au cours de l'été fait apparaître nettement l'augmentation d'évaporation de la zone irriguée par rapport à la Crau sèche. C'est ce qu'indique la figure 6 ; compte tenu des défaillances des systèmes de mesures notées plus haut, les résultats sont présentés séparément (Crau irriguée en 1978, Crau sèche en 1979), mais les 2 étés concernés ont été également secs et l'ordre de grandeur de rapports $\frac{\text { ETR }}{E T P}$ (correspondant ici au rapport des mesures $\frac{\mathrm{ET}_{\mathrm{BE}}}{\mathrm{ET}_{\mathrm{PE}}}$ ) sur des périodes de 3 à 4 mois $(0,8$ pour le premier cas, 0,4 dans le deuxième) apparaît comme représentatif du contraste entre les 2 zones.

\section{ESTIMATIONS DE L'ÉVAPORATION À PARTIR DES MESURES AU SOL}

Les résultats obtenus sur les stations de mesures au sol ont permis de comparer les méthodes faisant intervenir la thermographie IR à des méthodes classiques. 

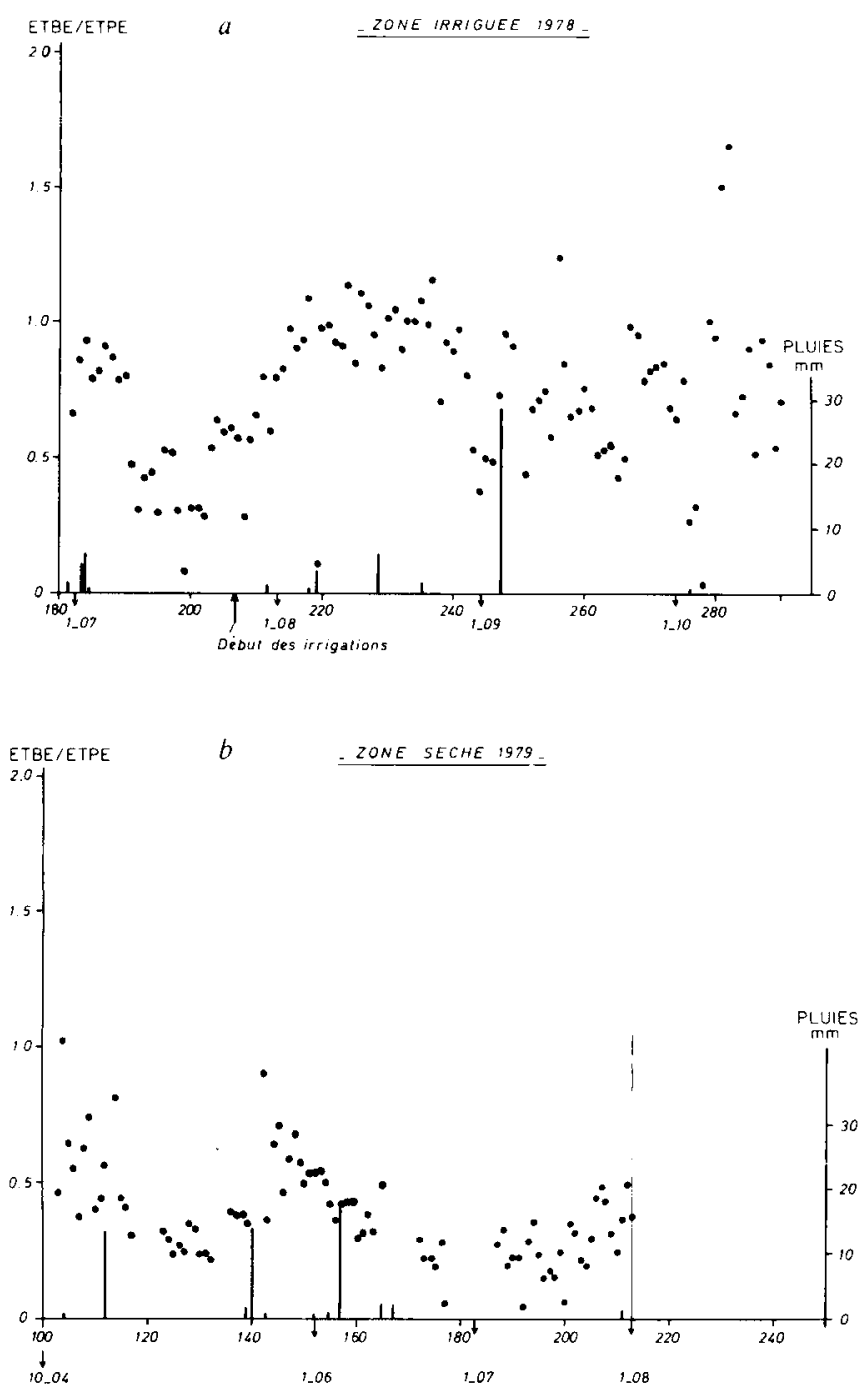

Figure 6

Evolution du rapport $\frac{E T_{B t}}{E T_{P t}}$

a) pour l'été 1978 en Crau irriguée.

b) pour l'été 1979 en Crau sèche.

Change in the ratio $\frac{E T_{B E}}{E T_{P t}}$

a) during summer 1978 in irrigated Crau.

b) during summer 1979 in dry Crau.

\section{A. Estimation de l'ETR en utilisant la température de surface $\mathbf{T}_{\mathrm{s}}$}

Cette estimation repose fondamentalement sur l'utilisation de l'équation du bilan d'énergie pour l'unité de surface homogène i (voir SEGUIN, 1979),

$$
\mathrm{ET}_{\mathrm{i}}=\mathrm{R}_{\mathrm{ni}}-\mathrm{S}_{\mathrm{i}}-\rho \mathrm{Cp} \mathrm{h}_{\mathrm{i}}\left(\mathrm{T}_{\mathrm{si}}-\mathrm{T}_{\mathrm{ai}}\right)
$$

où

$R_{n i}$ et $S_{i}$ sont respectivement le rayonnement net et le flux de chaleur dans le sol pour l'élément $\mathrm{i}, \mathrm{T}_{\mathrm{ai}}$ la température de l'air au-dessus de celui-ci et $h_{j}$ le coefficient d'échange turbulent entre la surface et la hauteur $\mathrm{z}$ de mesure de $\mathrm{T}_{\mathrm{ai}} \cdot \rho \mathrm{Cp}$ est une constante, correspondant au produit de la masse volumique et de la chaleur spécifique de l'air.

Pour évaluer les possibilités d'utiliser cette relation pour le calcul d'ETR, l'ET correspondante a été calculée, sur chaque site, au même pas de temps que les autres variables, en utilisant la mesure de $T_{s}$ par le radiothermomètre, ainsi que celle de $R_{n}$, $S$ et $T_{a}$.

Le point délicat réside dans l'estimation du coefficient d'échange h. Celui-ci s'exprime par l'ćquation suivante :

$h(\mathrm{z})=\frac{\mathrm{ku} *}{\log \frac{\alpha(\mathrm{z}-\mathrm{d})}{\mathrm{z}_{0}}} \mathrm{~F}_{2}\left(\frac{\mathrm{Z}}{\mathrm{L}}\right)$

u * étant la vitesse de frottement dérivée de la vitesse du vent $u(z)$ par la relation

$\mathrm{u}(\mathrm{z})=\frac{\mathrm{u} *}{\mathrm{k}} \log \frac{\mathrm{z}-\mathrm{d}}{\mathrm{z}_{0}} \mathrm{~F}_{1}\left(\frac{\mathrm{z}}{\mathrm{L}}\right)$

Dans ces 2 équations (2) et (3)

- $\mathrm{k}$ est la constante de VON KARMAN $(\sim 0,4)$

$-F_{1}\left(\frac{z}{L}\right)$ et $F_{2}\left(\frac{z}{L}\right)$ sont des fonctions de correction de stabilité atmosphérique traduite par la longucur de MONINOBUKHOV L. Nous avons adopté pour ces fonctions, les expressions les plus couramment admises à l'heure actuelle, en reprenant le calcul de SOER (1980) : celles formulées par PAULSON (1970) pour les conditions instables $\left(T_{s}>T_{a}\right)$ et

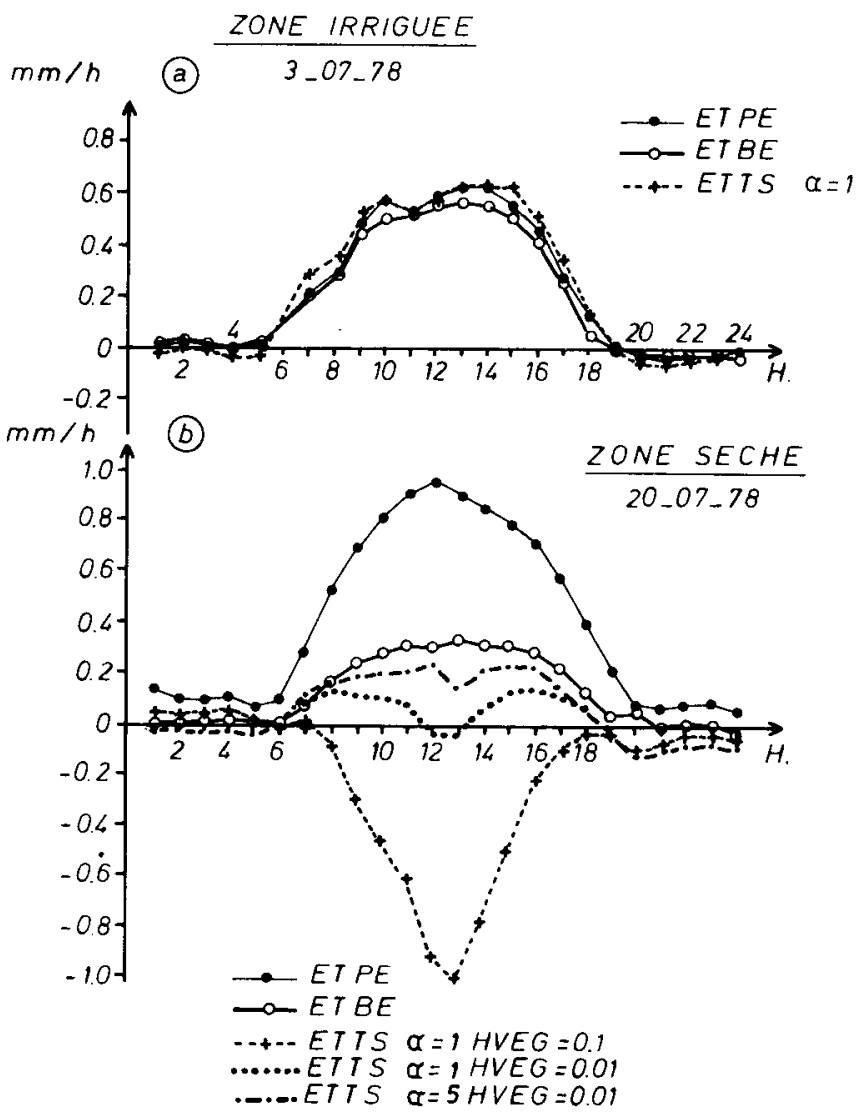

Figure 7

Evolution horaire des estimations des flux d'évaporation en zone irriguée (a) en Crau sèche (b). ET $T_{p t}=E T P$ par la formule de PENMAN; $E T_{B E}=E T$ mesurée par la méthode du bilan d'énergie; $E T_{I S}=E T$ estimée à partir de la méthode combinée avec $\alpha=1$ (sans correction) et $\alpha=5$ (correction de THOM) pour 2 hauteurs de végétation en zone sèche ( $H V E G=0,1 \mathrm{~m}$ et $H V E G=0,01 \mathrm{~m})$.

Hourly variation of evaporation fluxes for irrigated zone (a) and dry zone (b). ET $T_{P E}$ corresponds to PET by PENMAN formula; $E T_{B t}$ corresponds to measured ET using energy balance; $E T_{T S}$ to estimated ET using surface temperature Ts with $\alpha=1$ (without correction) and $\alpha=5$ (THOM correction) for two vegetation heights $(H V E G=0,1 \mathrm{~m}$ et $H V E G=0,01 \mathrm{~m})$. 
celles ćtablies par WEBB (1970) pour les conditions stables $\left(\mathrm{T}_{\mathrm{s}}<\mathrm{T}_{\mathrm{a}}\right)$.

- $\mathrm{z}_{0}$ et $\mathrm{d}$ sont les paramètres aérodynamiques du couvert de surface, respectivement équivalents à 0,10 et 0,66 HVEG (HVEG étant la hauteur de la végétation).

$-\alpha$ est un paramètre d'ajustement correspondant à la correction proposée par différents auteurs, dont THOM (1976), pour tenir compte de la non-similitude entre transferts de quantité de mouvement d'une part, de masse et chaleur d'autre part, au voisinage immédiat de surfaces rugueuses.

Nous avons considéré essentiellement 2 possibilités :

$\alpha=1$, correspondant à la non-prise en compte de cette correction et $\alpha=5$, suivant la recommandation formulée par THOM.

Le calcul d'ET $\mathrm{TS}_{\mathrm{TS}}$ par cette méthode, s'il est généralement satisfaisant en zone irriguée, fait apparaître de sérieux problèmes en zone sèche (fig. 7).

Aussi bien la valcur assignée au paramètre $Z_{0}$ (par le biais de la hauteur de végétation HVEG) que la prise en compte de la correction de Тном (passage de $\alpha$ de 1 à 5) ont un poids extrêmement important sur les valeurs prises par $\mathrm{ET}_{\mathrm{TS}}$. L'extrapolation spatiale d'ETR en utilisant la relation (1) se heurte donc, en premier lieu, à la nécessité de connaître avec précision le paramètre de rugosité $z_{0_{i}}$ de chaque unité, ce qui apparaît difficilement réalisable.

Dans le cas particulier de la Crau sèche, la valeur $Z_{0}=1 \mathrm{~mm}$ adoptéc a pu être vérifiée par la mesure de ce paramètre à partir de profils de vitesse du vent (estimations obtenues allant de 1 à $2 \mathrm{~mm}$ ). Pour la Crau irriguée, la valeur adoptée a été $Z_{0}=0,1 \mathrm{H}, \mathrm{H}$ étant la hauteur moyenne du couvert végétal estimée périodiquement. L'ensemble des calculs d'ET $\mathrm{TS}_{\mathrm{TS}}$ a donc été effectué avec ces valeurs de $Z_{11}$ et avec la correction de THOM $(\alpha=5)$.

\section{B. Estimation de l'ET journalière par la relation de JACKSON et al. (1977)}

En dehors des problèmes liés au poids du paramètre $Z_{0}$, l'estimation d'ET Ts par la relation (1) suppose, soit la mesure en continu de $\mathrm{T}_{\mathrm{s}}$ (ce qui n'est envisageable que dans le cas de satellites géostationnaires), soit l'extrapolation dans le temps à partir de mesures instantanécs correspondant aux passages du satellite.

Cette difficulté a amené JACKSON et al. (1977) à proposer l'estimation directe de l'ET journalière à partir de $R_{n}$ journalier et d'une valeur instantanée de l'écart $T_{s}-T_{a}$, de préférence au voisinage du maximum de température.

$$
\mathrm{ET}_{\mathrm{JT}}=\mathrm{R}_{\mathrm{nj}}-\mathrm{B}\left(\mathrm{T}_{\mathrm{s}}-\mathrm{T}_{\mathrm{a}}\right)
$$

L'utilisation de cette relation revient en fait à supposer :

1) qu'une valeur instantanée du flux de chalcur sensible $\mathrm{H}$ est suffisamment représentative, en moyenne sur un échantillon de plusicurs jours, du flux journalier,

2) que, dans les mêmes conditions de moyenne sur plusieurs jours, le coefficient d'échange $h$ peut être assimilé à une constante $\mathrm{B}$.

Des mesures préliminaires, effectuées sur une parcelle de gazon à Avignon au cours de l'année 1977, nous avaient permis d'établir (SEGUIN, 1979) que le principe de la procédure n'était pas irréaliste, mais que le principal problème résidait dans la connaissance du paramètre $B$, cclui-ci risquant de dépendre à la fois du type de climat et du

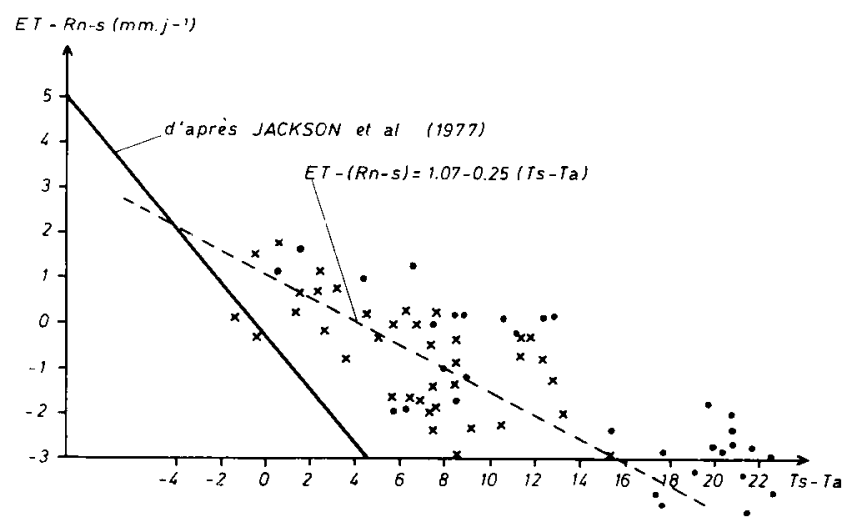

Figure 8

Relation entre ET - $\left(R_{n}-S\right)$ et $T_{S}-T_{a}$ pour 32 journées de l'été 78 sur le site de la Crau ( $\times$ Crau irriguée, - Crau sèche).

Relationship between ET- $\left(R_{n}-S\right)$ and $T_{S}-T_{n}$ for 32 days during summer 78 on the Crau site $(\times$ irrigated Crau, $\cdot$ dry Crau).

couvert végétal considéré. Une valeur beaucoup plus faible de $B$, en particulier, avait été obtenue (pour des résultats exprimés en $\mathrm{mm}^{-\mathrm{j}^{-1}}$ d'évaporation, de l'ordre de 0,05 contre 0,64 sur le blé à Phœnix dans l'expérience de JACKSON et al. ).

Il était donc intéressant d'utiliser les données de la Crau pour tester cette approche : c'est ce qui a été fait sur des mesures de l'été 78, en sélectionnant les journées où l'ensemble des mesures était disponible simultanément sur la zone sèche et la zone irriguée. 32 journées ont ainsi pu être traitées, les résultats obtenus étant reportés sur la figure 8 .

Il doit être précisé que la valeur d'ET pris en compte est la valeur de référence, déterminée par la méthode classique du rapport de BowEN et donc indépendante de la thermographie I.R.

L'examen de la figure 8 appelle plusieurs commentaires :

-- En premier lieu, sur le jeu de données disponibles, le bien-fondé de cette procédure simplifiée est confirmé. En dépit d'une dispersion inévitable (le coefficient de corrélation $r$ est égal à 0,67 ), l'utilisation de la droite de régression qui s'établit ainsi :

$$
\mathrm{ET}_{\mathrm{JT}}=\mathrm{R}_{\mathrm{n}}-\mathrm{S}+1,0-0,25\left(\mathrm{~T}_{\mathrm{s}}-\mathrm{T}_{\mathrm{a}}\right)
$$

(la valeur des flux étant expriméc en $m m^{-j^{-1}}$ ) permet l'approximation des valeurs individuelles journalières à $\pm 1,5 \mathrm{~mm}-\mathrm{j}^{-1}$.

Il faut noter que la relation obtenue fait intervenir une ordonnée à l'origine de $1,0 \mathrm{~mm}-\mathrm{j}^{-1}$, contrairement à celle établie par JACKSON et al. (1977), qui avaient introduit la condition de passage par ET $=0$ quand $T_{s}-T_{a}=0$. Cette condition ne nous semble pas obligatoire, dans la mesure où $\mathrm{T}_{\mathrm{s}}-\mathrm{T}_{\mathrm{a}}=0$ implique un flux de chaleur sensible $\mathrm{H}=0$ durant la journée, mais la période de nuit, à elle seule, peut effectivement introduire un écart entre ET et $R_{n}$ de l'ordre de $1 \mathrm{~mm}-\mathrm{j}^{-1}$.

- Le point intéressant à noter, pour cette relation, est qu'elle incorpore à la fois les données de la Crau sèche et de la Crau irriguée. Elle intègre donc non seulement une gamme de contraintes hydriques étendue, mais également 2 surfaces très différentes; un couvert continu herbacé de prairie de hauteur évoluant entre 10 et $50 \mathrm{~cm}$ au cours de la saison en zone irriguée, un sol nu caillouteux recouvert de végétation éparse et rase en zone sèche. 
Sans prétendre donc que la relation soit valable pour tous les types de couverts (cas des formations arbustives et des forêts, par exemple), il est au moins satisfaisant de constater qu'elle s'applique à ces 2 types de couverts.

- Enfin, les valeurs obtenues sont très différentes de celles de JACKSON et al. qui s'exprimaient, rappelons-le, par la relation $E T=R_{n}-0,64\left(T_{s}-T_{a}\right)$. Un tel écart peut s'expliquer par la variation de climat et de couvert végétal entre les 2 séries d'expériences. Une analyse approfondie des conditions d'expérimentation de Phœnix conduit cependant à penser que les valeurs rapportées par JACKSON et al. sont, en grande partie, influencées par des conditions d'advection locale résultant de la faible dimension des parcelles élémentaires $(12 \mathrm{~m} \times 90 \mathrm{~m})$. En particulier, la gamme d'écarts $\mathrm{T}_{\mathrm{s}}-\mathrm{T}_{\mathrm{a}}$ observés se situe dans le domaine de $T_{s}-T_{a}<0$, qui doit représenter une situation tout à fait exceptionnelle dans le cas des parcelles homogènes de grande étendue (de l'ordre minimal de $500 \mathrm{~m} \times 500 \mathrm{~m}$ ) repérables en télédétection à l'heure actuelle.

Il est donc possible de supposer que la relation établie sur les sites de la Crau serait beaucoup plus représentative des surfaces naturelles à prendre en compte pour la télédétection que celle de JACKSON et al., mais cette hypothèse demande à être vérifiée sur d'autres sites.

Quoi qu'il en soit, la relation (5) a ensuite pu être testée sur les séries de données précisées plus haut, qui constituent
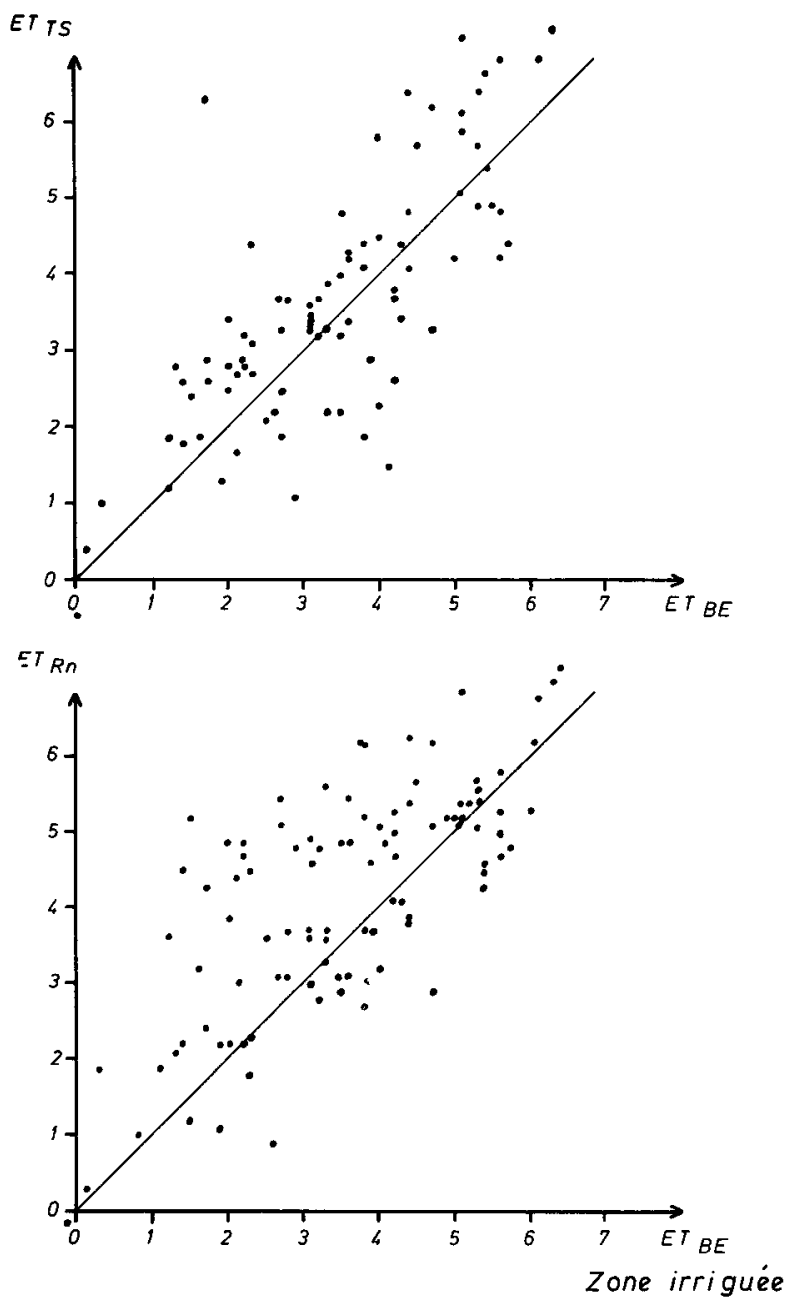

Figure 9

Comparaison des valeurs journalières obtenues par les différentes méthodes d'estimation a) $E T_{T S}$ b) $E T_{I T}$ c) $E T_{R N}$ d) $E T_{e q}$ pour 97 journées de l'été 78 en Z.I. un ensemble indépendant de celui sur lequel elles ont été établies.

\section{Comparaison entre les estimations d'ET basées sur la thermographie, les mesures de référence $\mathrm{ET}_{\mathrm{BE}}$ et des estimations simplifiées}

Pour établir, sur les sites de la Crau, la validité des 2 méthodes incorporant l'apport de la thermographie $\mathrm{ET}_{\mathrm{TS}}$ et $\mathrm{ET}_{\mathrm{JT}}$, les estimations journalières correspondantes ont été comparées aux valeurs de référence mesurées sur les sites par la méthode du bilan d'énergie $E T_{\mathrm{BE}}$.

Il est apparu, en outre, souhaitable d'effectuer la comparaison avec les estimations simplifiées qui peuvent être utilisées en hydrologie, de façon à réellement établir la portée de l'apport spécifique éventuel que l'on peut espérer de la télédétection. Dans cette optique, et en excluant le calcul d'ET par des modèles de bilan hydrique (qui supposent connues les réserves utiles RU des surfaces concernées, ainsi que les apports d'irrigation en Crau irriguée), deux approximations sont envisageables :

- soit $\mathrm{ET}=\mathrm{R}_{\mathrm{n}}$ (HLAVEK et al., 1974), reprise dans l'optique de la télédétection par VuILlaume et al. (1976), dont il est possible de penser, a priori, par l'analyse des connaissances courantes dans ce domaine, qu'elle risque
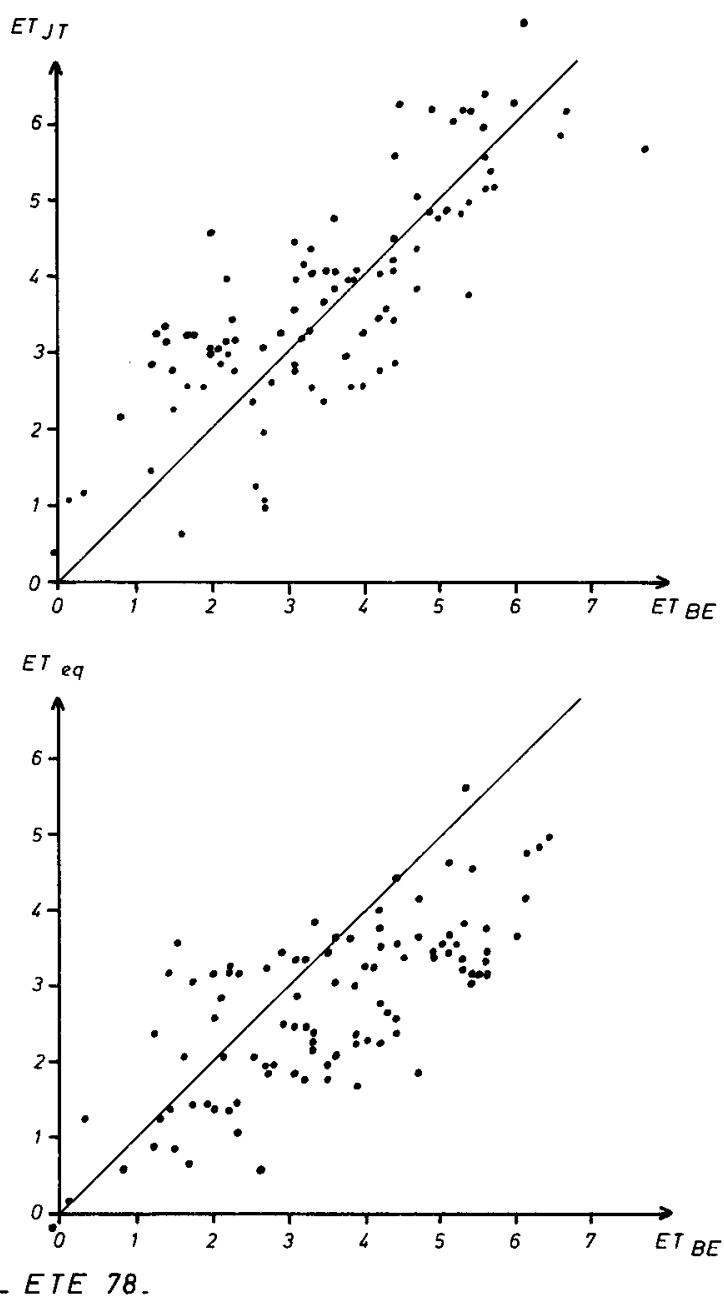

Comparison of daily values obtained by various methods a) $E T_{T S} b$ ) $E T_{J T}$ c) $E T_{R N}$ d) $E T_{e q}$ for 97 days during summer 78 in irrigated zone. 

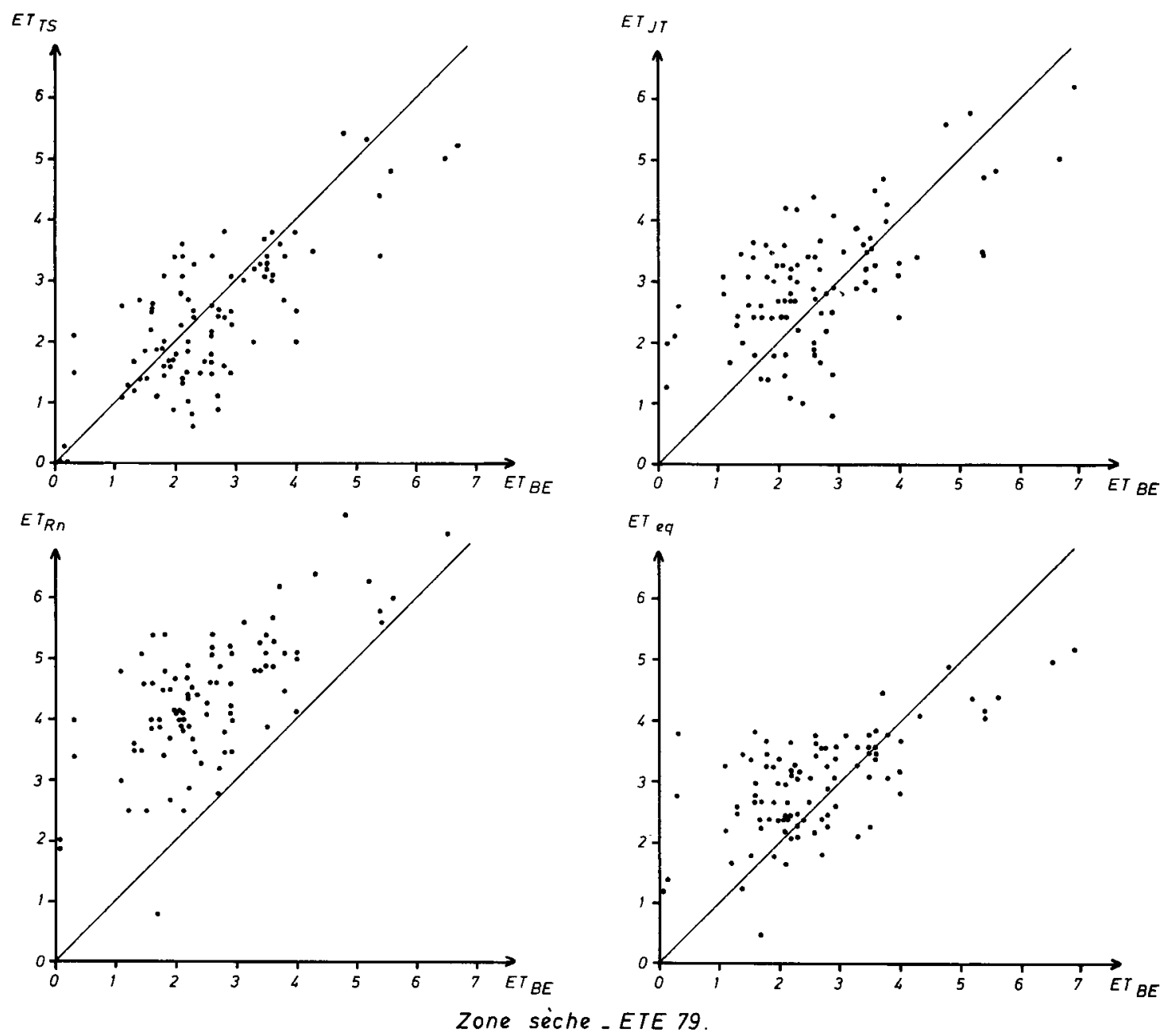

Figure 10

Zone séche - ETE 79

Comparaison des valeurs journalières obtenues par les différentes méthodes d'estimation a) $E T_{T S}$ b) $E T_{J T}$ c) $E T_{R N}$ d) $E T_{e q}$ pour 98 journées de l'été 79 en Z.S.

d'être satisfaisante dans des conditions proches de l'ETP, mais très surestimée en conditions sèches ;

- soit $\quad \mathrm{ET}=\mathrm{ET}_{\mathrm{cq}}=\frac{\Delta}{\Delta+\gamma} \mathrm{R}_{\mathrm{n}} \quad($ Perrier, 1977), qui correspond à la définition de l'«évaporation à l'équilibre », équivalente au $1^{\text {er }}$ terme de l'équation de Penman, et dont différents auteurs ont montré, ces dernières années, qu'elle constituait une approximation valable en conditions modérément sèches.

Ces 2 estimations, appelées respectivement $\mathrm{ET}_{\mathrm{RN}}$ et $\mathrm{ET}_{\mathrm{eq}}$, ont donc été introduites dans la comparaison portant sur les séries de l'été 78 en Crau irriguée et 79 en Crau sèche.

Les valeurs journalières obtenues par ces 4 méthodes d'estimation sont comparées aux valeurs de référence $\mathrm{ET}_{\mathrm{BE}}$ dans les figures 9 (été 78 Z.I.) et 10 (été 79 Z.S.).

$\mathrm{Au}$ vu de ces comparaisons, il apparaît :

- d'une part que les 2 méthodes basées sur la thermographie I.R. $\mathrm{ET}_{\mathrm{TS}}$ et $\mathrm{ET}_{\mathrm{JT}}$ donnent des résultats relativement satisfaisants, en dépit d'une dispersion assez élevée des valeurs individuelles, et ceci sur les 2 zones. Aucune des deux n'apparaît réellement plus performante que l'autre,

- d'autre part, pour les méthodes plus classiques, des résultats d'une précision comparable à celle de ces 2 méthodes peuvent être obtenus avec l'estimation $\mathrm{ET}=\mathrm{ET}_{\mathrm{RN}}$ en
Comparison of daily values obtained by various methods a) $E T_{T S} b$ ) $E T_{J T}$ c) $E T_{R N}$ d) $E T_{e q}$ for 98 days during summer 79 in dry Crau.

zone irriguée et $\mathrm{ET}=\mathrm{ET}_{\mathrm{cq}}$ en zone sèche. Par contre, l'approximation $\mathrm{ET}_{\mathrm{RN}}$ surestime nettement en zone sèche, alors que $\mathrm{ET}_{\mathrm{cq}}$ sous-estime en zone irriguée.

Ces appréciations peuvent être précisées et quantifiées par le calcul des valeurs cumulées sur l'ensemble de la période de mesures (les journées manquantes ayant été éliminées), ainsi que le montrent les figures 11 et 12 où ont été portées en plus les valeurs de l'ETP calculées par la formule de PENMAn à titre de référence.

Dans le cas de la zone irriguée, seule l'approximation $\mathrm{ET}_{J T}$ donne une précision satisfaisante sur le cumul (+12 p. 100). $E_{T S}$ dérive à partir de la moitié de la période de mesures pour aboutir à une surestimation de 20 p. 100 , comparable à celle de $\mathrm{ET}_{\mathrm{RN}}$, alors que $\mathrm{ET}_{\mathrm{eq}}$ sousestime nettement et de façon permanente $(-22$ p. 100).

Pour la zone sèche, aussi bien $\mathrm{ET}_{\mathrm{JT}} \mathrm{qu}$ 'ET $_{\mathrm{TS}}$ donnent des ordres de grandeur acceptables $(+13$ p. 100 et -8 p. 100 respectivement, ainsi qu'ET $(+16$ p. 100), alors qu'ET $T_{\mathrm{RN}}$ apparaît tout à fait inadapté.

Ces résultats chiffrés peuvent être déduits du tableau ciaprès, où sont présentées les valeurs cumulées sur l'ensemble de la période, ramenées en $\mathrm{mm}^{-\mathrm{j}^{-1}}$ à titre de comparaison.

$\begin{array}{lcccccc} & \mathrm{ETP} & \mathrm{ET}_{\mathrm{BE}} & \mathrm{ET}_{\mathrm{TS}} & \mathrm{ET}_{\mathrm{JT}} & \mathrm{ET}_{\mathrm{RN}} & \mathrm{ET}_{\text {eq }} \\ \text { Eté } 1978 \text { Z.I. } & 4,7 & 3,5 & 4,2 & 4,0 & 4,1 & 2,7 \\ \text { Eté 1979 Z.S. } & 6,7 & 2,6 & 2,4 & 2,9 & 4,4 & 3,0\end{array}$




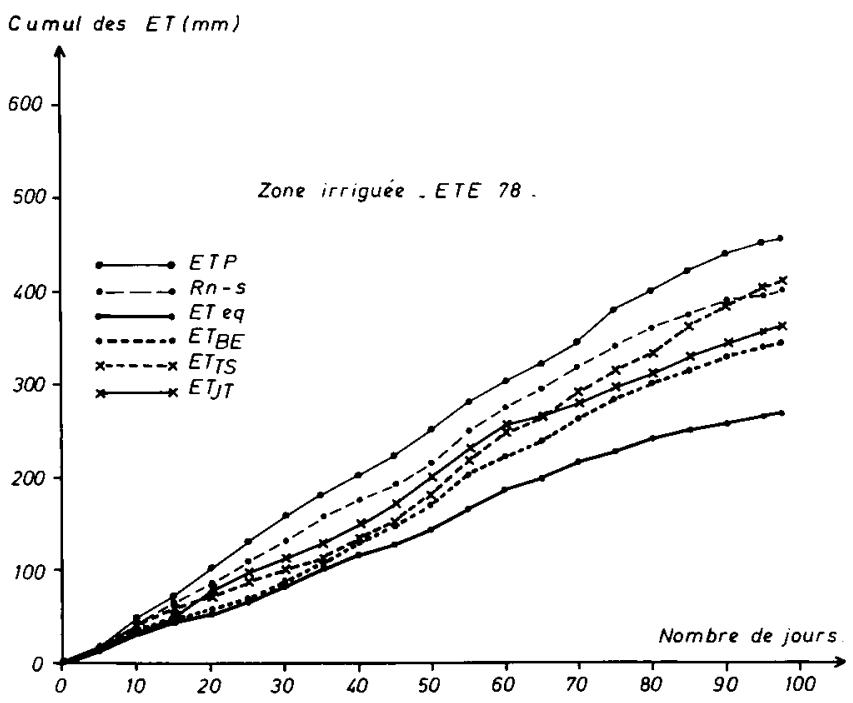

Figure 11

Cumul des valeurs d'évaporation sur la série journalière de l'été 78 en Z.I.

Summation of evaporation estimates during summer 78 in irrigated zone.

Ces résultats amènent les conclusions provisoires suivantes, valables évidemment uniquement pour le site de la Crau ct demandant à être confirmées par la suite.

- L'utilisation de la thermographie I.R. à l'aide d'un dispositif complémentaire important au sol permet d'obtenir une estimation satisfaisante de l'évaporation journalière, avec une précision de l'ordre de 10 à 15 p. 100 par rapport à la mesure de référence fournie par le bilan d'énergie. Il ne faut pas oublier que celle-ci, elle-même, ne peut raisonnablement pas être estimée avec une précision plus importante.

- Compte tenu des résultats comparables obtenus avec les 2 méthodes et dans une optique opérationnelle, la procédure simplifiée de JACKSON et al. (1977) apparaît beaucoup plus indiquée. L'estimation par le biais d'ET $\mathrm{TS}_{\mathrm{TS}}$, qui met en jeu une estimation continue des paramètres de rugosité $\mathrm{Z}_{0}$ de différentes surfaces difficilement envisageable en routine, n'apparaît pas réellement supérieure. Dans ces conditions, elle doit être considérée beaucoup plus comme outil méthodologique, pouvant servir de support à des modèles d'interprétation du type TELlus (RosEMA et al., 1978) que comme unc méthode opérationnelle.

Par contre, la méthode donnée par la procédure de JACKSON et al. pourrait déjà être utilisée opérationnellement sur le site de la Crau. Il reste, pour affirmer sa réelle valeur pratique, à évaluer la gamme de variation du coefficient B sur des surfaces naturelles variées et sous différents climats, en évitant d'effectuer les mesures correspondantes sur des surfaces de trop faibles dimensions.

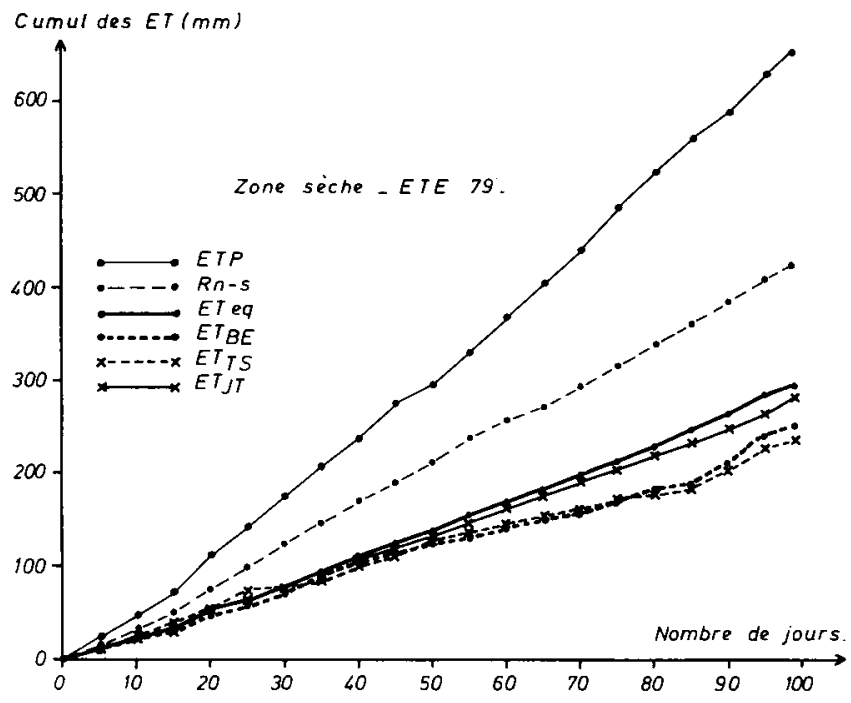

Figure 12

Cumul des valeurs d'évaporation sur la série journalière de l'été 79 en Z.S.

Summation of evaporation estimates during summer 79 in dry zone.

- De façon plus générale, le gain de précision apporté par la thermographie I.R. par rapport aux méthodes beaucoup plus simplifiées n'est pas énorme: 5 à 8 p. 100 d'amélioration par rapport à $\mathrm{ET}_{\mathrm{RN}}$ en zone irriguée et $\mathrm{ET}_{\mathrm{eq}}$ en zone sc̀che. Cependant, son gros avantage est de pouvoir s'appliquer à l'une et l'autre zone, alors que l'une ou l'autre des estimations $\mathrm{ET}_{\mathrm{RN}}$ et $\mathrm{ET}_{\mathrm{cq}}$ ne s'applique qu'à une seule situation. C'est donc le principal intérêt de la thermographie I.R. et son apport spécifique : s'appliquer avec le même ordre de grandeur de précision (10 à 15 p. 100) aux différentes situations, ce qui n'est pas le cas des approximations $E T=R_{n}$ tout à fait fausses en conditions sèches ou $E T=E_{c q}$ nettement insuffisantes en conditions irriguées.

Il reste à établir si cet avantage reste acquis lors du passage opérationnel à la télédétection, avec un minimum de mesures au sol. C'est le but d'un deuxième article qui sera publié ultérieurement.

Reçu le 15 avril 1981 Accepté le 16 septembre 1981.

\section{REMERCIEMENTS}

Les auteurs tiennent à exprimer leurs plus sincères remercicments à G. Thouy, R. Devillard et P. Reich, de la Station de Bioclimatologic I.N.R.A. d'Avignon, qui ont assumé respectivement l'installation et l'équipement des postes de mesure dans la Crau, leur entretien ainsi que le recueil des cassettes, puis leur lecture et leur traitement informatique.

\section{RÉFÉRENCES BIBLIOGRAPHIQUES}

Baelz S., 1980. Utilisation de thermographies infra-rouge recueillies par satellite pour le calcul de l'évapotranspiration à l'échelle régionale. Thèse de Doctorat de $3^{\mathrm{c}}$ cycle. Université de Paris VI. Sciences de l'Eau $119 \mathrm{p}$.

Hlavek R., Bakalowicz M., Oberlin G., Galea G., André R., Normand M., Oberlin S., 1974. Essai d'cstimation de l'ETR à l'échclle du bassin versant: considérations théoriques et applications pratiques. Bull. AIHS. 19 (4), 448-485.

Jackson R. D., Reginato R. J., Idso S. B., 1977. Wheat canopy temperature: a practical tool for evaluating water requirements. Water Resour. Res. 13 (3), 651-656.

Paulson C. A., 1970. The mathematical representation of wind 
speed and temperature profiles in the instable atmospheric surface layer. J. appl. Meteorol. 9 (1), 857-861.

Perrier A., Seguin B., 1970. Méthodes et techniques de détermination des coefficients de transport et des flux dans l'air. In "Techniques d'étude de facteurs physiques de la biosphère». I.N.R.A. ed., 425-445.

Perrier A., 1977. Projet de définitions concernant l'évapotranspiration en fonction de considérations théoriques et pratiques. La Météorologie VI" séric, $\mathrm{n}^{\prime \prime} 11$, Numćro spécial sur l'évapotranspiration, 7-16.

Petit V., 1980. Etude et modélisation de l'évapotranspiration réelle. Application au bilan hydrologique de la plaine de la Crau. Thèse de Doctorat de $3^{\text {e }}$ cycle. Université de Paris VI. Sciences de l'Eau, $102 \mathrm{p}$.

Rosema A., Bijleveld J. H., Reiniger P., Tassone G., Blyth K., Gurney R. J., 1978. "Tell-us », a combined surface temperature, soil moisture and evaporation mapping. 12th Int. Symp. Remote Sensing of Environ., Manille, Philippines, Avril 1978.

Seguin B., 1978. Modélisation des échanges d'énergie ct de masse à l'interface sol atmosphère. Problèmes posés par l'extension des modèles locaux à l'échelle régionale $(\sim 100 \mathrm{~km})$. C.R. Coll. natl. de l'ASP évolution des climats. Paris Dćc. 1978 F. BECKER ct C. PASTRE Ed. P. III.1 à III.19.

Seguin B., 1979. Application de la télédétection dans l'infra-rouge thermique à la détermination de l'évaporation et de l'humidité du sol. Cours FAO/OMM/ESA, Rome, Oct. 79. Ed. par l'ESA. SP $102,27-38$.

Seguin B., 1980. Détermination de l'ETR dans les bilans hydrologiques par la télédétection en thermographie infra-rouge. Bull. Sci. Hydr. 25 (2), 143-153.

Soer G. J. R., 1980. Estimation of the regional evapotranspiration and soil moisture conditions using remotely sensed crop surface temperature. Remote Sensing Environ. 9 (1), 27-47.

Thom A. S., 1976. Momentum, mass and heat exchange of plant commities. Dans "Vegetation and the Atmosphere". Ed. par MONTEITH J. L. Academic Press. London, Vol. 1, 57-109.

Vuillaume Y., Bossard R., Chenot D., 1976. Emploi du détecteur multispectral pour l'évaluation de l'évapotranspiration. Comm. pers. Journées du GDTA, Toulouse, Scpt. 1976, 15 p.

Webb E. K., 1970. Profile relationships: the log-linear range and extension to strong stability. Quart. J. Roy. Meteorol. Soc., 96, 6790. 